\title{
Low-power noncontact photoacoustic microscope for bioimaging applications
}

Krishnan Sathiyamoorthy

Eric M. Strohm

Michael C. Kolios 


\title{
Low-power noncontact photoacoustic microscope for bioimaging applications
}

\author{
Krishnan Sathiyamoorthy, ${ }^{\mathrm{a}, \mathrm{b}, \mathrm{c}}$ Eric M. Strohm, ${ }^{\mathrm{a}, \mathrm{b}, \mathrm{c}}$ and Michael C. Kolios ${ }^{\mathrm{a}, \mathrm{b}, \mathrm{c}, *}$ \\ ${ }^{a}$ Ryerson University, Department of Physics, Toronto, Ontario, Canada \\ 'Institute for Biomedical Engineering, Science and Technology (iBEST), Ryerson University and St. Michaels Hospital, Toronto, ON, Canada \\ 'Keenan Research Centre for Biomedical Science, St. Michael's Hospital, Toronto, Ontario, Canada
}

\begin{abstract}
An inexpensive noncontact photoacoustic (PA) imaging system using a low-power continuous wave laser and a kilohertz-range microphone has been developed. The system operates in both optical and PA imaging modes and is designed to be compatible with conventional optical microscopes. Aqueous coupling fluids are not required for the detection of the PA signals; air is used as the coupling medium. The main component of the PA system is a custom designed PA imaging sensor that consists of an air-filled sample chamber and a resonator chamber that isolates a standard kilohertz frequency microphone from the input laser. A sample to be examined is placed on the glass substrate inside the chamber. A laser focused to a small spot by a $40 \times$ objective onto the substrate enables generation of PA signals from the sample. Raster scanning the laser over the sample with micrometer-sized steps enables high-resolution PA images to be generated. A lateral resolution of $1.37 \mu \mathrm{m}$ was achieved in this proof of concept study, which can be further improved using a higher numerical aperture objective. The application of the system was investigated on a red blood cell, with a noiseequivalent detection sensitivity of 43,887 hemoglobin molecules $\left(72.88 \times 10^{-21}\right.$ mol or 72.88 zeptomol). The minimum pressure detectable limit of the system was $19.1 \mu \mathrm{Pa}$. This inexpensive, compact noncontact PA sensor is easily integrated with existing commercial optical microscopes, enabling optical and PA imaging of the same sample. Applications include forensic measurements, blood coagulation tests, and monitoring the penetration of drugs into human membrane. ( The Authors. Published by SPIE under a Creative Commons Attribution 3.0 Unported License. Distribution or reproduction of this work in whole or in part requires full attribution of the original publication, including its DOI. [DOI: 10.1117/1.JBO.22.4 .046001]
\end{abstract}

Keywords: microscope; photoacoustic; lasers; resolution; biomedical.

Paper 160343RRR received May 26, 2016; accepted for publication Mar. 7, 2017; published online Apr. 4, 2017.

\section{Introduction}

The photoacoustic (PA) effect was first observed in the year 1880 by Alexander Graham Bell. ${ }^{1}$ The PA effect initially was used in spectroscopy studies of materials throughout the mid1900 s, but it was not until invention of the laser that significant biomedical imaging applications using the PA technique became possible due to the improved sensitivity provided by laser excitation. $^{2-12}$ The low-power PA technique has been predominantly utilized in the field of spectroscopy, ${ }^{11-18}$ with substantial applications in trace gas detection. ${ }^{5,19-23}$ It exhibited significantly higher sensitivity than most other techniques, ${ }^{24-27}$ with concentration sensitivity in the parts per billion. ${ }^{3-5,25,27}$

PA spectroscopy is a powerful analytical tool for examining the optical absorption properties of solids as it directly measures the energy absorbed by the material on exposure to light. ${ }^{28}$ Conventional optical absorption/transmission spectroscopy requires a sample with a specialized surface as the scattering would significantly affect the accuracy of the measurement of optical absorption coefficient. It cannot be used to study highly scattering samples. ${ }^{29}$ The only other method of obtaining spectroscopic information from opaque samples is diffused optical reflection spectroscopy, where special handling is required for sample surface preparation. In the case of PA spectroscopy, spectral information can be obtained from a variety of samples,

*Address all correspondence to: Michael C. Kolios, E-mail: mkolios@ryerson .ca including opaque materials such as powders, metals, and semiconductors. ${ }^{2,12}$

The principle and theory of the standard PA technique were well established by Rosencwaig, who described the fundamental principles and investigated several possible applications of the PA technique. ${ }^{30}$ Patel and Tam reviewed the physics of the technique and also extended the technique to other applications such as liquids. ${ }^{31-33}$

Several types of PA cells have been proposed by various investigators. ${ }^{4,19,31,34-37}$ PA cells made of a kilohertz microphone and piezoelectric transducers (PZT) have been widely studied. ${ }^{31,34,37,38}$ Gas-microphone PA cells were generally used to study powder and samples with large surface to volume ratio, whereas PZT PA cells were used for liquid and bulk solids. ${ }^{2}$ The gas-microphone PA cell possesses a relatively better sensitivity at low modulation frequencies. ${ }^{2}$ The sample in the gas-microphone PA cell is held in the air tight sample metal chamber and is excited through the optical window. Light absorption by a material leads to two types of de-excitation processes: radiative and nonradiative. The nonradiative de-excitation results in heating the sample. Since the incident radiation is intensity modulated by the chopper, the sample gets heated repetitively. The resulting periodic heat flow from the solid absorber to the surrounding gas creates pressure fluctuations in the cell, which are detected by the microphone. Therefore, the depth of specimen responsible for the PA signal is restricted within a thermal diffusion length defined by ${ }^{30}$ 
$\mu=\frac{k}{\pi \rho f c}$,

where $k$ is the thermal conductivity, $\rho$ is the density, $c$ is the specific heat, and $f$ is the chopping frequency. The depth profiling of the sample is possible by varying the chopping frequency. ${ }^{39-41}$ Thomas et al. ${ }^{42}$ detected subsurface flaws in aluminum in the PA magnitude to a depth of approximately one diffusion length ( $\sim 180$ to $800 \mu \mathrm{m}$ ) over a wide range of chopping frequencies. Other than spectral measurements, the PA technique can also be used in several other applications such as the measurement of thermal diffusivity, ${ }^{43}$ detection of phase transitions, ${ }^{44}$ and luminescence quantum efficiency. ${ }^{45}$ The present aim is to use this PA spectroscopy principle for photoacoustic microscopy (PAM) imaging applications.

PAM is a hybrid imaging modality that uses an optical technique for excitation and an acoustic technique for detection. In the conventional approach, the acoustic wave is generated by transient pressure variation caused by the absorption of a nanosecond light pulse. High-resolution deep tissue imaging is possible using acoustic resolution PA imaging. ${ }^{46-49}$ PA imaging has been used to image anatomical, ${ }^{50}$ functional, ${ }^{46}$ molecular, ${ }^{46}$ flow dynamic, ${ }^{47}$ and metabolic contrasts in vivo. ${ }^{47}$ Microscopy applications can use either acoustic resolution PAM (AR-PAM) or optical resolution PAM (OR-PAM), or a combination of both, depending on the application and imaging target. ${ }^{51}$ The lateral resolution of AR-PAM depends on the acoustic focus of the transducer, which would be on the order of tens of micrometers $^{49}$ with a maximum imaging depth of several millimeters. The lateral resolution of OR-PAM depends on the diffraction limited spot size produced by the lens with a maximum depth imaging of $1.2 \mathrm{~mm} .^{52,53}$ OR-PAM was used to image small animal models and biological samples with various endogenous or exogenous contrasts. ${ }^{52}$ AR-PAM is a promising imaging technique for both preclinical and clinical applications as deep imaging of biological tissues possible with lower frequency transducers. ${ }^{53}$

Conventional PA imaging systems require expensive ultrafast lasers with nanosecond pulse widths, an ultrasound transducer, and an aqueous coupling medium for the PA waves to propagate through. ${ }^{49,51}$ Clinical adoption has been hindered due to its expense and cumbersome size. Considerable efforts have been made to use low-cost continuous wave (CW) lasers instead of ultrafast lasers for PA imaging. ${ }^{54,55}$ Petschke and La Rivière theoretically investigated the possibility of using chirped CW diode lasers, ${ }^{55}$ which would exhibit 20 to $30 \mathrm{~dB}$ lower SNR than the typical pulsed laser-based systems, but its compactness and relatively low cost could potential outweigh the lower SNR in selected applications. ${ }^{54-56}$

Other noncontact PA systems based on the interferometric principle have been developed. ${ }^{57-61}$ However, interferometric techniques were complex, bulky, expensive, and difficult to integrate with the existing optical microscopes. ${ }^{57,62}$ A Fabry-Perot sensor is another option, but it is still a contact technique as the Fabry-Perot sensor has to be placed in contact with the sample. ${ }^{10,63}$ Researchers have investigated using a fiber sensor, which also required expensive focusing and collection optics. Optical beam deflection techniques are another simple method for PA wave detection. However, this approach requires two lasers for pumping and probing and a large footprint area for the set up because the probe beam needs to travel a relatively long distance (for increasing deflection angle) before detection to improve sensitivity. ${ }^{64,65}$ There are also other air couple transducers available, but air-coupled transducers are unfocused, bulky, and exhibit poor SNR, which makes imaging more complicated. ${ }^{66}$

This study is aimed at developing a low-cost air-coupled PA imaging system using common low-power CW lasers and a kilohertz-range microphone, using the PA spectroscopy principle. In the PA spectroscopy, a wide area of the sample is illuminated to get spectroscopy information of aggregates. The typical PA cell used for spectroscopy cannot be used for high-resolution OR-PAM application as the sizes (in the range of a few millimeter to a few inches) and design of the chamber limits the focusing of light. ${ }^{12,23,35,36}$ We also developed PA cell for spectroscopy studies. The PA cell was made using a bulk aluminum rod to avoid vibrational noise. The powdered sample was taken in a sample holder made of copper and enclosed in an air tight metallic PA chamber with a thick optical window above the sample. The developed-PA cell was suitable for an epi-illumination excitation, where the sample was excited over the surface by passing the light through an optical window and sample chamber (SC) air column. The sample was excited over a wide area $(6 \mathrm{~mm})$ to get the PA spectrum. A thick optical window was used to dampen the vibration noise. A high-magnification objective could not be used in this configuration for a high-resolution PA image due to the short working distance.

In this study, we designed a PA chamber suitable for a lowpower CW laser-based high-resolution PAM. The PA cell was designed to fit into existing optical microscopes for multimodal imaging. As the PA spectroscopy technique can be used to study samples in any state such as solid, liquid, and gas, ${ }^{19,33,34}$ numerous applications exist, such as coagulation tests to study clotting disorders like thrombophilia and hemophilia, blood pattern analysis (BPA) in forensics, ${ }^{67}$ and monitoring the penetration of drugs into membrane acceptor systems. ${ }^{68}$

\section{Materials and Methods}

\subsection{Experimental Setup}

A dual modal optical/acoustic microscope system was developed as shown in Fig. 1(a). The optical system is built as an inverted microscope configuration consisting of a halogen lamp, an infinity-corrected $40 \times$ and 0.6 NA Olympus objective (O), a mirror (M5), and a Ximea xiQ camera (Ximea). The system consists of motorized linear $X-Y$ scanning stages (Zaber Technologies) for raster scanning and manual $X-Y-Z$ stages (Thorlabs) for manual adjustment. A diode pumped solid state green laser (532 nm, CrystaLaser, Nevada) was used as a source of PA excitation. The laser beam diameter was $0.36 \mathrm{~mm}$ and operated at $3 \mathrm{~mW}$ power. The beam waist size at the sample surface was $1.2 \mu \mathrm{m}$. The beam was intensity modulated for PA generation using a mechanical chopper. The laser path in the system was described by the solid green line in Fig. 1(a). The light beam at the beam splitter is split into two beams, one for the sample and the other for the reference. The reference beam was monitored by a photodiode and used to normalize the PA signal to avoid any change due to laser fluctuations. The reflected light from the beam splitter was focused on the sample using the objective. As the light beam was periodically intensity modulated using a chopper, the periodic pressure wave was created in the PA cell which was measured using a lock-in amplifier. All components were computer controlled through custom MATLAB software (Mathworks). The lock-in amplifier is 

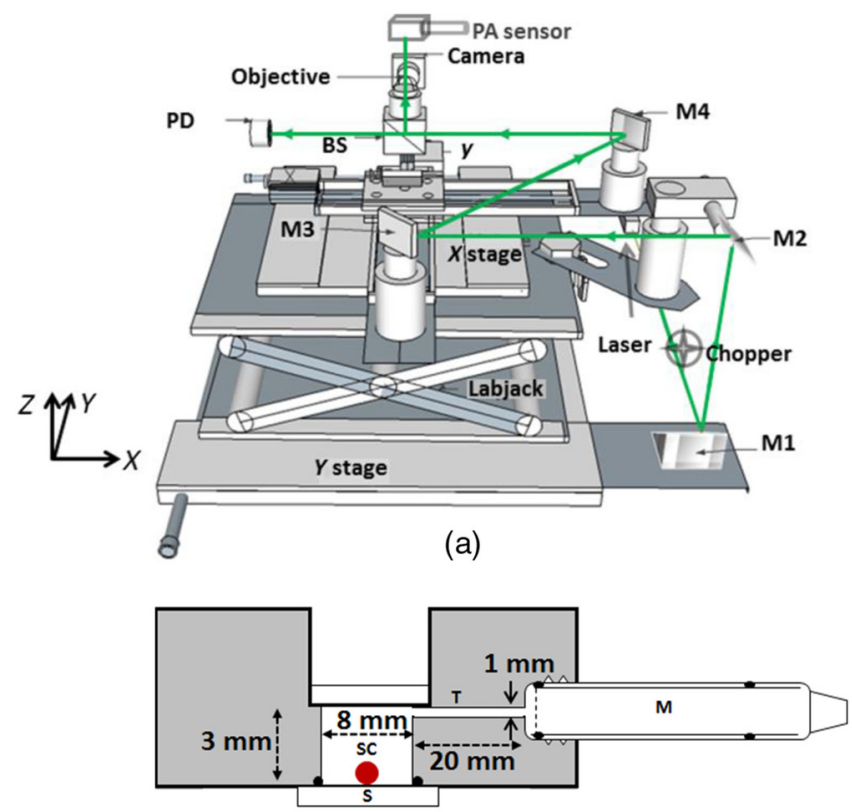

(b)

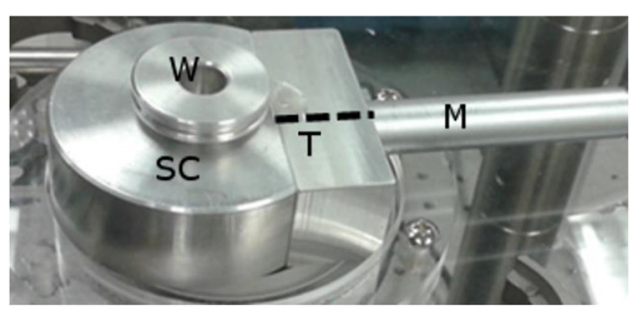

(c)

Fig. 1 (a) A Schematic diagram of a dual model system that contained optical and PA microscopes. (The solid green line represents the beam propagation direction), (b) the schematic diagram of the PA sensor, and (c) photograph of the PA sensor. M1, M2, M3, and M4 are mirrors, $\mathrm{M}$ is the microphone, $\mathrm{SC}$ is the sample chamber, $\mathrm{W}$ is the window, and $\mathrm{T}$ is the resonator tunnel.

operated at low-pass filter with a single RC filter $(6 \mathrm{~dB} /$ oct roll off) with a time constant of $300 \mathrm{~ms}$. An equivalent bandwidth $(\Delta f)$ of a lock-in is $1 / 4 T$, where $T$ is the time constant $(R \times C)$. In this study, $\Delta f$ is equal to $2.5 \mathrm{~Hz}$. To scan the image of size $0.3 \mathrm{~mm} \times 0.3 \mathrm{~mm}$ by $60 \times 60$ steps would take up to $20 \mathrm{~min}$. This long scan time is due to the slow serial communications interface used. Future enhancements use a USB interface to increase the scan time by $10 \times$.

\subsection{Photoacoustic Resonance Chamber}

The PA cell used in the PA spectrometer was modified for the PAM applications. ${ }^{2,12,35,36}$ The PA cell was developed to use high-magnification objectives for sample excitation. The PA cell was designed in a double optical window configuration for easy integration into existing optical systems. The top of the PA cell contained a thick glass window and the bottom contained a glass substrate to hold the sample. As the sample is excited from the bottom through the glass substrate, high-magnification objectives can be used. It can be integrated both in epi and trans-illumination commercial optical microscopes for multimodal imaging, including both optical and PA imaging. Since the sample was heated at the glass-sample interface, thermal diffusivity properties of the sample and backing material (glass) will play an important role in the PA signal generation. The backing material reduces the PA generation by diffusing the heat generated in the sample by the laser away from the chamber. The thermal diffusion length and viscous-thermal damping distance of air will also play important roles in PA generation due to the smaller size of PA cell. The SC size was designed based on the thermal diffusion length and viscous-thermal damping distance of air to prevent the acoustic damping loss at the glass window and the thermo-viscous damping loss at the chamber walls. The thermal diffusion length and viscousthermal damping of gas are dependent on frequency, temperature, and pressure. At room temperature and pressure, the air thermal diffusion length and viscous-thermal damping distances are 0.2 and $0.1 \mathrm{~mm}$, respectively, at a frequency $100 \mathrm{~Hz} .{ }^{12}$ A cell that is designed to be used over a wide range of frequencies should then have a minimum distance between the sample and window and minimum passageway dimensions of 1 to $2 \mathrm{~mm} .{ }^{12}$ The PA cell was built in a Helmholtz configuration, a chamber with an acoustic resonator. The resonator helps to isolate the microphone from the scattered light in the SC, which would improve the SNR. ${ }^{69}$

Figures 1(b) and 1(c) show the schematic and picture of this PA sensor. The cell was made from an aluminium rod of diameter $80 \mathrm{~mm}$. Aluminium was used as it exhibits a large thermal mass, which would result in minimal PA signal generation. The PA sensor contains two compartments, one for the sample (SC) and the other for the microphone (M). The SC is cylindrical with an 8-mm diameter and 3-mm height. The top of the SC is closed by an optical window made of BK7 glass and the bottom of the chamber holds the sample (S). A small resonant column (T) exists between the SC and the microphone (M). The resonant column is a long cylindrical column of 20-mm length and 1-mm diameter. The microphone used as the PA detector has a frequency response of 50 to $20 \mathrm{kHz}$ and a sensitivity of $50 \mathrm{mV} / \mathrm{Pa}$ (Brüel\&Kjær Sound and Vibration Measurement, Pointe-Claire, Quebec, Canada). The laser was chopped using a mechanical chopper (SR540, Stanford Research Systems), which has a chopping frequency between 50 to $3000 \mathrm{~Hz}$. The laser was modulated at $200 \mathrm{~Hz}$ in the present studies. The generated PA signal was measured using a lock-in amplifier (SR850, Stanford Research Systems), which is interfaced with the computer through an RS232 port.

\subsection{Procurement of Blood}

Blood was collected by netCAD (Vancouver, Canada), the research division of Canadian Blood Services, under protocol 2013-001, which involves standard Canadian Blood Services collection and testing procedures of whole blood and delivery overnight at $4^{\circ} \mathrm{C}$, with continuous monitoring during shipment to ensure no temperature deviations occur. This procedure has been approved by the research ethics boards of both Ryerson University and Canadian Blood Services.

\subsection{Blood Sample Preparation}

The guidelines on handling the blood were followed in accordance with the recommendations of the International Society for Clinical Hemorheology and the European Society for Clinical Hemorheology and Microcirculation. The blood was centrifuged at room temperature at $2000 \times g$ for $6 \mathrm{~min}$ to separate the plasma and their Buffy coat. Isotonic phosphate buffered saline (PBS) was used to wash RBCs two times. The centrifuged RBCs were then dispersed in PBS for the present studies. 


\subsection{Lateral Resolution Measurement}

The system lateral resolution was determined using a USAF 1951 resolution test chart (Thorlabs). The 1951 USAF resolution test chart consists of reference line patterns with welldefined thicknesses and spacing. The positive targets consist of low-reflectivity, vacuum-sputtered chrome patterns plated on clear substrates. These targets have 6 groups $(+2$ to +7$)$ with six elements, offering a maximum resolution of 228.0 line pairs per millimeter. The thickness of each chrome line was $0.12 \mu \mathrm{m}$. The spatial resolution of the PA system was evaluated by measuring the PA signal across an element 2 of group 6 , which has a linewidth $(6.96 \mu \mathrm{m})$ close to the size of a single RBC size.

\subsection{Modulation Transfer Function}

The modulation transfer function (MTF), which is commonly used to evaluate optical systems, is another way to evaluate the resolution of the system. The MTF describes contrast transfer from the object to image intensity. The image quality mainly depends on the aberration of the optical system and pixel resolution of the camera. The contrast (MTF) was obtained by measuring the signal from the bright $\left(I_{\max }\right)$ and the dark bars $\left(I_{\min }\right)$. The contrast ratio was calculated using the expression

$\%$ contrast $=\frac{I_{\max }-I_{\min }}{I_{\max }+I_{\min }} * 100$,

where $I_{\max }$ and $I_{\min }$ are the PA intensities in the presence and absence of the sample. The USAF resolution target is generally used to calculate the MTF of optical microscope systems, where the dark bar is the metal strip and the bright bar is the transparent region between the bars.

The PA signal produced was proportional to the thermal diffusion length of the sample and surroundings, which would be a few hundred nanometers to a few microns depending on the thermal property of the materials. The thermal diffusion to the surrounding medium would result in blurring the PA image, and, therefore, would affect the resolution of the system. The USAF resolution chart would not provide the exact MTF of the bioimaging system as it is made of metal, which has different physical properties (thermal, acoustical, and optical) than biological samples. The present system would be used to image the internal features of biological cells, which have their own physical properties, characteristic shape, and relative positions.
Hence, the MTF of the system was also calculated using the RBCs. The MTF of the optical system was calculated from the sinusoid pattern with a decrease in the spatial frequencies. The spatial frequency of contrast $0.5(50 \%)$ was regarded as the minimum detectable contrast limit of the system. ${ }^{70,71}$ Therefore, a monolayer of RBCs distributed at various spacing on a glass substrate is more representative of the real-world applications of this imaging system.

\subsection{Noise Equivalent Sensitivity and Minimum Detectable Pressure}

The molecular sensitivity of the system was evaluated in terms of a minimum number of hemoglobin molecules detectable by the system, as it is one of the most common target chromophores in PA imaging. ${ }^{72}$ A single RBC contains about $270 \times 10^{6}$ hemoglobin molecules. The mean corpuscular volume is $92.8 \times 10^{-15} \mathrm{~L}$. The estimated concentration of hemoglobin in a single RBC is $3.1 \times 10^{6} \mu \mathrm{m}^{-3}{ }^{72}$ The typical diameter of normal RBCs was 6 to $8 \mu \mathrm{m}$ and its thickness was 2 to $2.5 \mu \mathrm{m}$. The total hemoglobin molecules excited by light focused using a $40 \times$ and 0.6 NA objective with an input light beam of diameter of $0.36 \mathrm{~mm}$ was $7 \times 10^{6}$.

The noise equivalent number of molecules $(\mathrm{NEM})=$ no of illuminated molecules/SNR.

The SNR was calculated by dividing an average PA signal from an RBC by a standard deviation of the background noise. The average PA signal was obtained by irradiating an $\mathrm{RBC}$ over a time period of $5 \mathrm{~min}$. The minimum pressure detectable limit (MPDL) of the sensor was then estimated. The MPDL $=$ standard deviation of the background noise/sensitivity of the microphone.

\section{Results and Discussion}

Figures 2(a)-2(c) show the optical image, the PA image, and the corresponding line spread function (LSF), respectively, of a single element in the USAF test target.

The LSF of the system was obtained by numerically differentiating an edge spread function (ESF, solid black line). The ESF represents an average value obtained from the line strip marked by a yellow rectangular box in the PA image 2(b). The lateral resolution limit of the system was calculated from the full width half maximum (FWHM), which was obtained using a Gaussian fit to the LSF. The solid red line in Fig. 2(c) is the Gaussian fit to the LSF. From this measurement, the system is determined to have a lateral resolution of $2.1 \mu \mathrm{m}$.

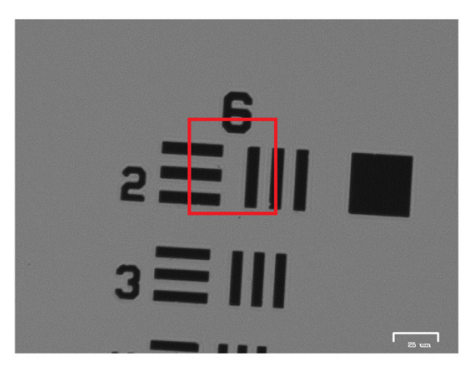

(a)

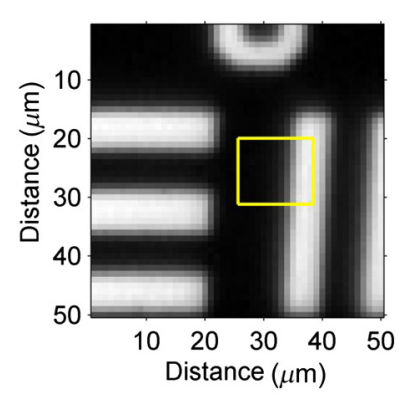

(b)

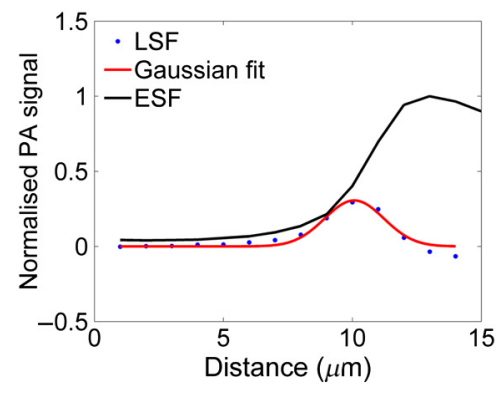

(c)

Fig. 2 Spatial resolution measurement: (a) optical image of group-6 of the USAF-1951 chart, (b) PA image of element-2 of group- 6 (marked by red square in optical image), (c) ESF (black line) and the LSF (blue dots) calculated from PA signal in the rectangular box of the PA image. The solid red line represents the Gaussian fit to the LSF. 
Measurements were performed on a common blood smear on a glass slide to probe single RBC. The system was operated with the settings described in Sec. 2.1. Figure 3 shows the optical and PA images of the RBCs.

The MTF of the system was calculated using the USAF chart and an RBC monolayer. The MTF measurement from element 2 of group 6 of USAF chart demonstrated a $76 \%$ contrast ratio. Figure 4(a) shows the PA image of the RBCs. Line profiles $\mathrm{AB}, \mathrm{CD}$, and $\mathrm{EF}$ were drawn across $\mathrm{RBCs}$ that exhibit various separations between them. The PA line profiles were obtained [Figs. 4(b)-4(d)] along these lines. The valley (1 to 5) in the line profile represents the separation whose value was obtained from FWHM of the Gaussian fit. The MTF was calculated along those lines by measuring the signal from two neighboring RBCs and the signal from the gap between them. The signal from the RBC was obtained by averaging signals measured across RBCs, whereas the signal from the gap was obtained by averaging signal above the FWHM. Table 1 shows the FWHM of separation of RBC pairs 1 to 5 and their contrast ratios, which were calculated using the expression 2 .

The contrast of the PA image as optical image decreased with an increase in spatial frequency (Table 1). The RBCs at spacing $2.16 \mu \mathrm{m}$ exhibited contrast close to PA image of element 2 group 6 of the USAF chart even though its physical properties such as shape, optical, and thermal were completely different. The lowest separation that the system could resolve was $1.37 \mu \mathrm{m}$, which was close to the interrogation spot size (waist size $=1.2 \mu \mathrm{m}$ ) of the laser beam. The separation of the $\mathrm{RBCs}$ at position 1 could not be resolved as the spacing was
Table 1 MTF calculation of system at various RBC aggregates.

\begin{tabular}{lcc} 
Position & FWHM $(\mu \mathrm{m})$ & MTF $(\%)$ \\
\hline 1 & Unresolvable & - \\
2 & 2.16 & 39.86 \\
3 & 2.96 & 65.55 \\
4 & 1.89 & 20.37 \\
5 & 1.37 & 6.27 \\
\hline
\end{tabular}

$<1.2 \mu \mathrm{m}$. The resolution measurement using the USAF 1951 test target was higher, probably due to the larger thermal diffusivity $\left(29 \times 10^{-6} \mathrm{~m}^{2} / \mathrm{s}\right)$ and effusivity of chrome.

Next, the noise equivalent sensitivity (equivalent to molecular sensitivity) of the system was calculated. The average PA signal obtained from the RBC due to a point excitation by a $40 \times$ objective was $9.66 \times 10^{-5} \mathrm{~V}$, whereas the standard deviation of the background noise was $9.54 \times 10^{-7} \mathrm{~V}$. Therefore, the SNR of this measurement is $\sim 100$. As the signal is generated from $\sim 7 \times 10^{6}$ hemoglobin molecules (see Sec. 2.7), the NEM is $\sim 693 \times 10^{2}$.

The NEM per unit bandwidth $(\mathrm{NEM} / \sqrt{\Delta f})$ is $\sim 43,887 \mathrm{~Hz}^{-1 / 2}$ or $72.88 \times 10^{-21} \mathrm{~mol}$ or 72.88 zeptomol. The system exhibited a sensitivity of nearly half of the value reported by Winkler et al. ${ }^{72}$ The sensitivity was due to an increase in the

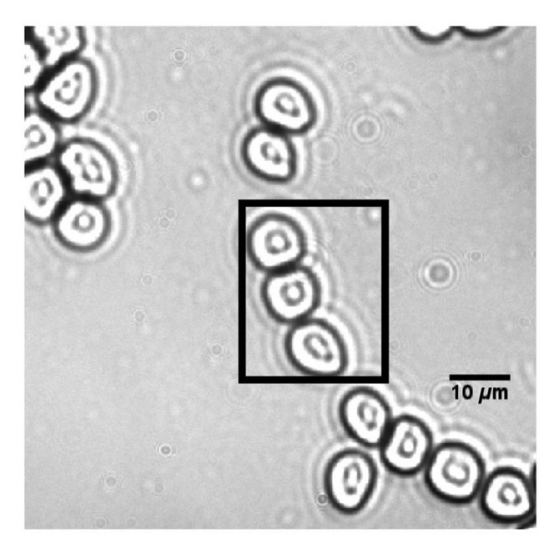

(a)

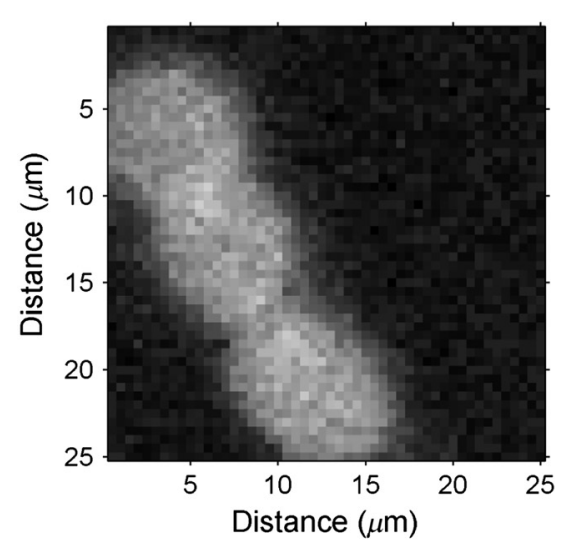

(b)

Fig. 3 (a) Optical and (b) PA images of RBC (marked in a black rectangular box in the optical image) smeared on a glass substrate.

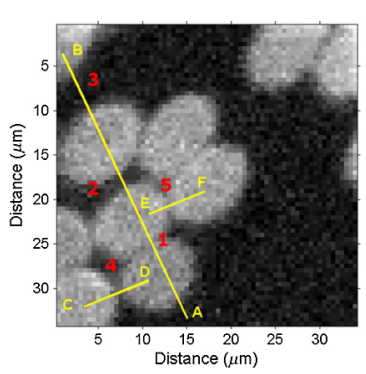

(a)

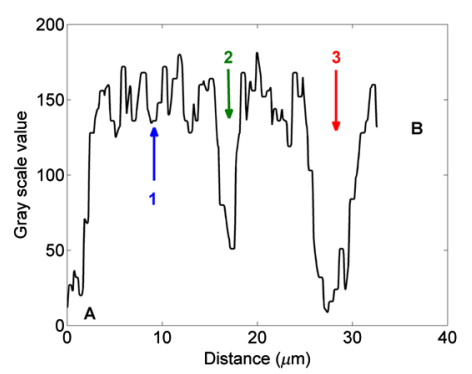

(b)

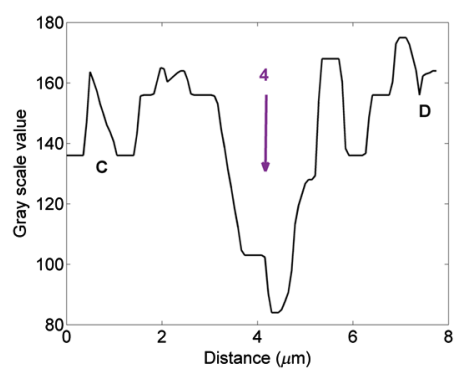

(c)

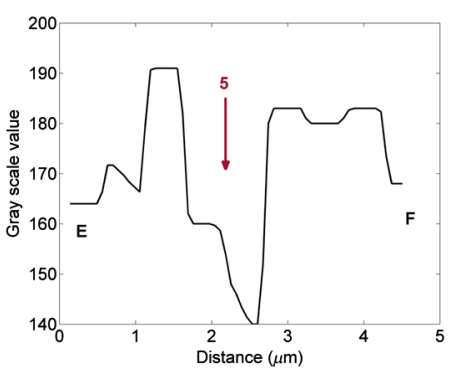

(d)

Fig. 4 (a) PA image of the RBC monolayer, (b) PA line profile measured along a line AB marked in PA image, (c) PA line profile measured along a line CD, and (d) PA line profile measured along a line EF. 
PA energy due to integration time of the lock-in amplifier. The sensitivity can be improved further by increasing the integration time, but would increase the image acquisition time by reducing the scanning rate.

Next, the MPDL of the sensor was estimated. The average PA signal generated by $7 \times 10^{6}$ hemoglobin molecules was $9.66 \times 10^{-5} \mathrm{~V}$, and, in the same measurement, the standard deviation of background noise was $0.954 \mu \mathrm{V}$. The sources for this noise were the amplifier input noise, the Johnson noise of the resistor, and shot noise due to nonuniform flow of current charge carrier. If we assume that all the sources of noise are incoherent and random in nature, then the total noise can be estimated using the square root of the sum of the squares of all the noise. Since the sensitivity of the microphone is $\sim 50 \mathrm{mV} / \mathrm{Pa}$, we estimate the MPDL to be $19.1 \mu \mathrm{Pa}$.

Amplifier input noise and Johnson noise were Gaussian nature; the amount of noise measured was proportional to the square root of the bandwidth of the lock-in amplifier. Hence, the MPDL per unit bandwidth, MPDL $/ \sqrt{\Delta f}=$ $7.64 \mu \mathrm{Pa} / \mathrm{Hz}^{1 / 2}$.

Other PAM systems require a fluid coupling medium for PA signal detection. ${ }^{46-49,51,61,63}$ This system uses air as a coupling medium and, thus, can be used to image samples that cannot normally be imaged using PAM, such as samples that would be altered or destroyed through contact with fluids, including powders or water-soluble dyes. There are other noncontact techniques based on optical interferometers such as Mach-Zehnder interferometer, ${ }^{57,58}$ low-coherence Michelson interferometer, ${ }^{59}$ and Fabry-Perot interferometer. ${ }^{61,63}$ These systems are typically complex, expensive, and difficult to integrate with another system. In most cases, the sample surface also required special treatment as the scattering and diffuse surface of the sample would affect the reflection of the probe light (sample arm) of the interferometer system and thereby its SNR. The FabryPerot sensor can also be used, but it is a contact technique as the sensor has to be placed in contact with the sample. ${ }^{57,62}$ Our sensor is inexpensive, simple, and easy to integrate with existing optical microscopes. The sensor can be used for opaque and highly scattering samples. As the microphone is located far from the laser beam direction, optical imaging techniques such as epi-fluorescence, confocal, Raman, phase contrast, bright, and dark field microscopy can be performed on the sample.

\section{Conclusions}

This study demonstrates a compact noncontact PAM system using a low-power CW laser source for PA measurements. Air can be used as the coupling fluid, enabling PA measurements of any sample type. The system exhibits a lateral resolution of $1.37 \mu \mathrm{m}$ and a noise equivalent hemoglobin molecular sensitivity of 43,887 molecules $\left(72.88 \times 10^{-21} \mathrm{~mol}\right.$ or 72.88 zeptomol). Future work will focus on improving the sensitivity and the resolution using high numerical aperture objectives and measurements using different CW laser wavelengths, enabling an inexpensive, high-resolution, multispectral PAM system. The compactness of the PA sensor will enable easy integration with existing commercial optical microscopes for multimodal imaging, with numerous biomedical and forensic applications.

\section{Disclosures}

The authors have no financial interests in this work.

\section{Acknowledgments}

This work was funded by the Natural Sciences and Engineering Research Council of Canada (No. 216986-2012) and Ryerson University. Funding to purchase the equipment was provided by the Canada Foundation for Innovation, the Ontario Ministry of Research and Innovation, and Ryerson University.

\section{References}

1. A. G. Bell, "On the production and reproduction of sound by light," Am. J. Sci. 20(118), 305-324 (1880).

2. A. Rosencwaig, Photoacoustics and Photoacoustic Spectroscopy, John Wiley \& Sons Inc., New York (1980).

3. E. L. Kerr and J. G. Atwood, "The laser illuminated absorptivity spectrophone: a method for measurement of weak absorptivity in gases at laser wavelengths," Appl. Opt. 7(5), 915 (1968).

4. L. B. Kreuzer, "Ultralow gas concentration infrared absorption spectroscopy," J. Appl. Phys. 42(7), 2934-2943 (1971).

5. L. B. Kreuzer, N. D. Kenyon, and C. K. N. Patel, "Air pollution: sensitive detection of ten pollutant gases by carbon monoxide and carbon dioxide lasers," Science 177(4046), 347 (1972).

6. F. Müller et al., "Transportable, highly sensitive photoacoustic spectrometer based on a continuous-wave dualcavity optical parametric oscillator," Opt. Express 11(22), 2820-2825 (2003).

7. A. Danielli et al., "Label-free photoacoustic nanoscopy," J. Biomed. Opt. 19(8), 086006 (2014).

8. M. Xu and L. V. Wang, "Photoacoustic imaging in biomedicine," Rev. Sci. Instrum. 77(4), 41101 (2006).

9. B. Cox et al., "Quantitative spectroscopic photoacoustic imaging: a review," J. Biomed. Opt. 17(6), 061202 (2012).

10. P. Beard, "Biomedical photoacoustic imaging," Interface Focus 1(4), 602-631 (2011).

11. A. Rosencwaig, "Photoacoustic spectroscopy," Ann. Rev. Biophys. Bioeng. 9, 31-54 (1980).

12. A. Rosencwaig, "Photoacoustic spectroscopy of solids," Opt. Commun. 7(4), 305-308 (1973).

13. J. B. Kinney and R. H. Staley, "Applications of photoacoustic spectroscopy," Annu. Rev. Mater. Sci. 12(1), 295-321 (1982).

14. G. A. West et al., "Photoacoustic spectroscopy," Rev. Sci. Instrum. 54(7), 797-817 (1983).

15. C. Haisch, "Photoacoustic spectroscopy for analytical measurements," Meas. Sci. Technol. 23(1), 012001 (2012).

16. K. Sathiyamoorthy, C. Vijayan, and M. P. Kothiyal, "Simple technique for obtaining photoacoustic spectra corrected for the spectral variation of the source in single scan," Rev. Sci. Instrum. 78(4), 043102 (2007).

17. D. V. Bageshwaret, "Photoacoustic spectroscopy and its applicationsa tutorial review," 187-203 (2010).

18. J. Li, W. Chen, and B. Yu, "Recent progress on infrared photoacoustic spectroscopy techniques," Appl. Spectrosc. Rev. 46(6), 440-471 (2011).

19. P. C. Claspy, "6-infrared optoacoustic spectroscopy and detection A2-PAO, YOH-HAN," in Optoacoustic Spectroscopy and Detection, pp. 133-166, Academic Press, New York (1977).

20. L. B. Kreuzer, "1-the physics of signal generation and detection A2-PAO, YOH-HAN," in Optoacoustic Spectroscopy and Detection, pp. 1-25, Academic Press, New York (1977).

21. M. B. Robin, "7-photoacoustic spectroscopy of gases in the visible and ultraviolet spectral regions A2-PAO, YOH-HAN," in Optoacoustic Spectroscopy and Detection, pp. 167-191, Academic Press, New York (1977).

22. C. K. N. Patel, E. G. Burkhardt, and C. A. Lambert, "Spectroscopic measurements of stratospheric nitric oxide and water vapor," Science 184(4142), 1173 (1974).

23. P. C. Claspy et al., "Laser optoacoustic detection of explosive vapors," Appl. Opt. 15(6), 1506-1509 (1976).

24. C. R. Philbrick, "Remote sensing of chemical species in the atmosphere," in Proc. Fourth Symp. Lidar Atmospheric Application (2009).

25. T. Kobayashi, "Techniques for laser remote sensing of the environment," Remote Sens. Rev. 3(1), 1-56 (1987).

26. A. Elia et al., "Photoacoustic techniques for trace gas sensing based on semiconductor laser sources," Sensors 9(12), 9616-9628 (2009). 
27. C. M. Wynn et al., "High-sensitivity detection of trace gases using dynamic photoacoustic spectroscopy," Opt. Eng. 53(2), 021103 (2013).

28. A. Rosencwaig and J. B. Willis, "Photoacoustic absorption measurements of optical materials and thin films," J. Appl. Phys. 51(8), 4361-4364 (1980).

29. L. W. Burggraf and D. E. Leyden, "Quantitative photoacoustic spectroscopy of intensely light-scattering thermally thick samples," Anal. Chem. 53(6), 759-764 (1981).

30. A. Rosencwaig and A. Gersho, "Theory of the photoacoustic effect with solids," J. Appl. Phys. 47(1), 64-69 (1976).

31. C. K. N. Patel and A. C. Tam, "Pulsed optoacoustic spectroscopy of condensed matter," Rev. Mod. Phys. 53(3), 517-550 (1981).

32. C. K. N. Patel and A. C. Tam, "Optoacoustic spectroscopy of liquids," Appl. Phys. Lett. 34(7), 467-470 (1979).

33. A. C. Tam and C. K. N. Patel, "Optical absorptions of light and heavy water by laser optoacoustic spectroscopy," Appl. Opt. 18(19), 33483358 (1979).

34. A. Rosencwaig, "Photo-acoustic spectroscopy of solids," Rev. Sci. Instrum. 48(9), 1133-1137 (1977).

35. W. G. Ferrell and Y. Haven, "High-performance cell for photoacoustic spectroscopy," J. Appl. Phys. 48(9), 3984-3985 (1977).

36. D. Cahen, E. I. Lerner, and A. Auerbach, "Simple setup for single and differential photoacoustic spectroscopy," Rev. Sci. Instrum. 49(8), 1206-1209 (1978).

37. A. Rosencwaig and G. Busse, "High-resolution photoacoustic thermalwave microscopy," Appl. Phys. Lett. 36(9), 725-727 (1980).

38. C. F. Dewey, Jr., "3-design of optoacoustic systems A2-PAO, YOHHAN," in Optoacoustic Spectroscopy and Detection, pp. 47-77, Academic Press, New York (1977).

39. J. Opsal and A. Rosencwaig, "Thermal-wave depth profiling: theory," J. Appl. Phys. 53(6), 4240-4246 (1982).

40. G. F. Kirkbright et al., "Depth-resolved spectroscopic analysis of solid samples using photoacoustic spectroscopy," Anal. Chem. 56(12), 2043 2048 (1984).

41. J. W. Nery et al., "Photoacoustic spectroscopy for depth-profile analysis and herbicide monitoring in leaves," Analyst 112(11), 1487-1490 (1987).

42. R. L. Thomas et al., "Subsurface flaw detection in metals by photoacoustic microscopy," J. Appl. Phys. 51(2), 1152-1156 (1980).

43. J. Joseph et al., "Thermal diffusivity variations in nanoparticle administered phantom tissues- a photoacoustic investigation," Eur. Phys. J. Appl. Phys. 5930501 (2012).

44. Q. Shen, M. Inoguchi, and T. Toyoda, "The influence of chemical postetching and UV irradiation on the optical absorption and thermal diffusivity of porous silicon studied by photoacoustic technique," in Proc. Sixth Int. Conf. Nano-Molecular Electronics (ICNME 2004), pp. 161-167 (2006).

45. M. J. Adams, J. G. Highfield, and G. F. Kirkbright, "Determination of the absolute quantum efficiency of sodium salicylate using photoacoustic spectroscopy," Analyst 106(1265), 850-854 (1981).

46. H. F. Zhang et al., "Functional photoacoustic microscopy for highresolution and noninvasive in vivo imaging," Nat. Biotech. 24(7), 848-851 (2006).

47. J. Yao et al., "High-speed label-free functional photoacoustic microscopy of mouse brain in action," Nat. Meth. 12(5), 407-410 (2015).

48. E. M. Strohm, E. S. L. Berndl, and M. C. Kolios, "High frequency labelfree photoacoustic microscopy of single cells," Photoacoustics 1(3-4), 49-53 (2013).

49. J. Yao and L. V. Wang, "Photoacoustic microscopy," Laser Photonics Rev. 7(5), 758-778 (2013).

50. Y. Zhang et al., "Non-invasive multimodal functional imaging of the intestine with frozen micellar naphthalocyanines," Nat. Nanotechnol. 9(8), 631-638 (2014).

51. E. M. Strohm, M. J. Moore, and M. C. Kolios, "Single cell photoacoustic microscopy: a review," IEEE J. Sel. Top. Quantum Electron. 22(3), 137-151 (2016).
52. L. Zhu et al., "Multiview optical resolution photoacoustic microscopy," Optica 1(4), 217-222 (2014).

53. S. Park et al., "Acoustic resolution photoacoustic microscopy," Biomed. Eng. Lett. 4(3), 213-222 (2014).

54. K. Maslov and L. V. Wang, "Photoacoustic imaging of biological tissue with intensity-modulated continuous-wave laser," J. Biomed. Opt. 13(2), 024006 (2008).

55. A. Petschke and P. J. La Rivière, "Comparison of intensity-modulated continuous-wave lasers with a chirped modulation frequency to pulsed lasers for photoacoustic imaging applications," Biomed. Opt. Express 1(4), 1188-1195 (2010).

56. T. Wang et al., "A low-cost photoacoustic microscopy system with a laser diode excitation," Biomed. Opt. Express 5(9), 3053-3058 (2014).

57. S. A. Carp and V. Venugopalan, "Optoacoustic imaging based on the interferometric measurement of surface displacement," J. Biomed. Opt. 12(6), 064001 (2007).

58. A. Hochreiner et al., "Non-contact photoacoustic imaging using a fiber based interferometer with optical amplification," Biomed. Opt. Express 4(11), 2322-2331 (2013).

59. Y. Wang, C. Li, and R. K. Wang, "Noncontact photoacoustic imaging achieved by using a low-coherence interferometer as the acoustic detector," Opt. Lett. 36(20), 3975-3977 (2011).

60. J. Eom, S. J. Park, and B. H. Lee, "Noncontact photoacoustic tomography of in vivo chicken chorioallantoic membrane based on allfiber heterodyne interferometry," J. Biomed. Opt. 20(10), 106007 (2015).

61. E. Zhang, J. Laufer, and P. Beard, "Backward-mode multiwavelength photoacoustic scanner using a planar Fabry-Perot polymer film ultrasound sensor for high-resolution three-dimensional imaging of biological tissues," Appl. Opt. 47(4), 561-577 (2008).

62. B. P. Payne et al., "Optoacoustic tomography using time-resolved interferometric detection of surface displacement," J. Biomed. Opt. 8(2), 273-280 (2003).

63. P. C. Beard, F. Perennes, and T. N. Mills, "Transduction mechanisms of the Fabry-Perot polymer film sensing concept for wideband ultrasound detection," IEEE Trans. Ultrason. Ferroelectr. Freq. Control 46(6), 1575-1582 (1999).

64. R. A. Barnes et al., "Probe beam deflection technique as acoustic emission directionality sensor with photoacoustic emission source," Appl. Opt. 53(3), 511-519 (2014).

65. J. L. Johnson et al., "Gas-coupled laser acoustic detection as a noncontact line detector for photoacoustic and ultrasound imaging," J. Opt. 18(2), 024005 (2016).

66. R. G. M. Kolkman et al., "Feasibility of noncontact piezoelectric detection of photoacoustic signals in tissue-mimicking phantoms," J. Biomed. Opt. 15(5), 055011 (2010).

67. N. Laan et al., "Bloodstain pattern analysis: implementation of a fluid dynamic model for position determination of victims," Sci. Rep. 5, 11461 (2015).

68. B. D. Hanh et al., "Penetration of compounds through human stratum corneum as studied by Fourier transform infrared photoacoustic spectroscopy," J. Controlled Release 70(3), 393-398 (2001).

69. J. F. McClelland and R. N. Kniseley, "Scattered light effects in photoacoustic spectroscopy," Appl. Opt. 15(12), 2967-2968 (1976).

70. T. L. Willimas, B. A. Leach, and B. J. Biddles, "A workshop instrument for testing binocular and other sights using the m.t.f. criterion," Opt. Laser Technol. 4(3), 115-120 (1972).

71. K. Murata, "IV instruments for the measuring of optical transfer functions," in Progress in Optics, Vol. 5, E. Wolf Ed., pp. 199-245, Elsevier (1966).

72. A. M. Winkler, K. Maslov, and L. V. Wang, "Noise-equivalent sensitivity of photoacoustics," J. Biomed. Opt. 18(9), 097003 (2013).

Biographies for the authors are not available. 